\title{
Modelo de análisis multicriterio en la evaluación de los diferentes cultivos para producción de biodiesel
}

Multicriteria analysis model in the evaluation of different crops for biodiesel production

\author{
Juan Fernandes Herrera Guardiola, Rafaela Ferreira Lopes, \\ João Carlos Correa Baptista Soares de Mello e Flávio Castro da Silva
}

\author{
Universidade Federal Fluminense, RJ, Brasil \\ juanfernando@id.uff.br; rafaelafl@id.uff.br; joaocsmello@gmail.com; flavio-ter@vm.uff.br
}

\begin{abstract}
Resumo
El modelo de Macbeth es una herramienta multicriterio para la toma de decisiones que se ha utilizado en diferentes proyectos y estudios de las energías renovables. El propósito de este trabajo fue aplicar el software Macbeth para la evaluación delos cultivos de canola, maíz, palma y soya, teniendo en cuentacritérios técnicos, ambientales y económicos y sus respectivos pesos. El cultivo de canolaresultó ser la mejor opción para la producción de biodiesel, con un global de 74 puntos debido a su buena puntuación en criterios ambientales la cual tenía un mayor peso comparado com los otros criterios.El cultivo de maíz fue el segundo colocado, presentando buenos resultados en todos los criterios, seguido del cultivo de Palma. La última posición la ocupó el cultivo de soya con 36,67 puntos en este análisis.
\end{abstract}

Palabras clave: Biocombustibles; Energías renovables; MCDA; Macbeth

\begin{abstract}
Macbeth model is a multicriteria tool for the multicriteria decision making that has been used in different projects and studies of renewable energies. The purpose of this paper was apply the software Macbeth for the evaluation of canola, corn, palm and soy crops taking in to account technician, environmental and economic criterias and their weights. Canola crop was the best option for the biodiesel production, with a global score of 74 points because of their good score in environmental criterias, wich had a higher weight than the other criterias. Corn crop was the second positioned, presenting good results in all criterias, followed by palm crop. The final one was soy crop with 36,67 points in this analysis.
\end{abstract}

Keywords: Biofuels; Renewable energies; MCDA; Macbeth 


\section{Introducción}

En los últimos años la producción de biocombustibles ha aumentado debido, entre otras razones, al aumento de la demanda energética causada por el crecimiento demográfico, a la escasez de reservas de hidrocarburos, incremento de precios de combustibles a partir de fuentes fósiles, y a los impactos ambientales producidos por este tipo de combustibles.

Como alternativa a estas problemáticas en diferentes países, entre ellos las tradicionales potencias agrícolas como Brasil, Estados Unidos y parte de la Unión Europea, han invertido en el desarrollo de diferentes biocombustibles que sean menos contaminantes y derivados de fuentes renovables (CRAGO et al., 2010). Brasil, de acuerdo con la Asociación Internacional Renewable Energies21, fue el segundo mayor productor de biocombustibles con una producción total de 30.8 billones de litros, donde la producción de etanol fue de 27 billones de litros y de biodiesel 3.8 (SAWIN y SEYBOTH K., 2017).

Por otro lado, la Dirección de Biocombustibles de la Unión Europea exigió a sus Estados miembros lograr para el 2020 que el 10\% de la matriz energética sea de biocombustibles en el mercado de combustibles líquidos según la Comisión Europea (2007), citado por (ABUNHABAYA et al., 2012).

Entre estos biocombustibles se encuentra el biodiesel que ha sido uno de los más utilizados en diferentes países- entre ellos Brasil por su alta diversidad biológica (CUNHA et al., 2009) - debido a su rápida y fácil producción a partir de numerosas fuentes renovables tales como aceites vegetales, animales y de microalgas.

Además, la densidad de este biocombustible es similar al diésel derivado de petróleo, permitiéndole ser mezclado con facilidad y ser utilizado en motores diésel sin ninguna modificación mecánica. También, el biodiesel es considerado biodegradable, no toxico, buen lubrificante para las partes del motor (ANGUEBES et al., 2016), así como prácticamente no contener azufre, produciendo menos cantidad de gases efecto invernadero por unidad de energía utilizable y contribuyendo significativamente en la reducción de impactos ambientales de acuerdo con Mota (2009) citado por (DE CASTRO, 2013).

No obstante, el crecimiento en la producción de biocombustibles requiere de grandes áreas agrícolasespecíficamente para la plantación de oleaginosas- y otros recursos para la producción de las materias primas generando grandes impactos ambientales, entre ellos la seguridad alimentar.

Por este motivo, es necesario encontrar procesos de producción de biocombustibles cada vez más sustentables teniendo en cuenta sus impactos sociales, técnicos, políticos y ecológicos. Debido a estos aspectos la herramienta análisis multicriterio (MCDA por sus siglas en inglés), es necesaria para seleccionar el cultivo más sustentable para la producción de biodiesel. En este orden de ideas, el objetivo del presente trabajo es determinar, mediante el análisis multicriterio, el cultivo que sea más adecuado para la producción de biodiesel.

\subsection{Análisis multicriterio}

La metodología MCDA es utilizada para resolver problemas que involucran conflictos múltiples y emergentes, teniendo prioridades o agregando pesos de importancia a cada criterio dado por el "tomador de decisiones".

El método MCDA ha sido utilizado en diversas ocasiones para resolver problemas de la vida diaria, donde se cuenta con varias alternativas de acuerdo con los criterios que involucran la toma de decisiones. Unas de las aplicaciones de MCDA han sido en análisis de políticas energéticas, planeación de plantas eléctricas, selección de tecnologías y evaluación de proyectos y análisis de impactos ambientales (STRANTZALI AND y ARAVOSSIS, 2016).

La aplicación del método MACBETH fue desarrollada por Bana e Costa y Chagas en 2004, atendiendo a cuestiones esenciales tales como que cada criterio se relaciona a una escala de valores a través de calificaciones atribuidas para cada alternativa, necesitando ser transformada en cuantitativa cuando la evaluación es cualitativa. El método utiliza siete categorías cualitativas de atracción, definiendo así la preferencia del decisor.

Dadas dos alternativas del modelo, el decisor debe comparar las opciones e informar si es indiferente o 
si la diferencia es muy leve, leve, moderada, fuerte, muy fuerte o extrema. El uso exclusivo de juicios cualitativos es el gran diferencial de MACBETH (BANA E COSTA y CHAGAS, 2004).

Posteriormente, los niveles de desempeño son comparados entre sí donde al final para cada alternativa, de acuerdo al criterio analizado, se le atribuye un valor al nivel de desempeño.

Específicamente en proyectos de energías renovables, técnicas de MCDA han sido empleadas y presentadas por (HARALAMBOPOULOS y POLATIDIS, 2003), (DIAKOULAKI y KARANGELIS, 2007), (COBULOGLU y BÜYÜKTAHTAKIN, 2015), (KURKA y BLACKWOOD, 2013), entre otros.

Inclusive Strantzali y Aravossis en 2016, revisaron las metodologías utilizadas para la modelación de sistemas de energías renovables durante las últimas décadas y encontró que las más aplicadas fueron el análisis de ciclo de vida (LCA por sus siglas en ingles), análisis de costo beneficio (CBA por sus siglas en ingles) y MCDA.

Por otro lado, Andrade et al. (2016), analizaron 8 tipos de energías tanto convencionales (no renovables) como alternativas (renovables), y las comparó teniendo en cuenta criterios técnicos, financieros, ambientales y socio-económico-políticos, mediante la metodología multicriterio Macbeth, obteniendo como resultado prevalencia en las energías renovables y favoreciendo al uso de energías más sustentables.

De igual forma Chaves y Gomes (2014) evaluaron diferentes biocombustibles de acuerdo a aspectos económicos, ambientales, sociales y técnicos mediante el método Macbeth, encontrando el bioetanol como la opción más sustentable entre los biocombustibles analizados.

Según Cobuloglu y Büyüktahtakin (2015), la sustentabilidad para cultivos generadores de energías a partir de su biomasa están enmarcados e integrados en 3 dimensiones: la dimensión económica que considera los rendimientos de conversión a biocombustible de los cultivos, eficiencia acumulada, costos operacionales de los cultivos, tecnologías y ciclo fenológico del cultivo. La segunda dimensión ambiental tiene en cuenta la erosión del suelo producida por cada cultivo, captura del dióxido de carbono, biodiversidad y contaminación de agua generada por el cultivo. Finalmente, la dimensión social consiste en el mejoramiento del bienestar social, tasa de desempleo y condiciones de trabajo.

Strantzali y Aravossis (2016), encontraron que los principales criterios utilizados en la planeación de proyectos de energía son: técnicos, económicos, ambientales y sociales. Así mismo determinó que los subcriterios más utilizados son el costo de inversión del proyecto y emisiones de $\mathrm{CO}_{2}$, seguido de la generación de empleo como enfoque en el ámbito social. Ya en el área técnica, se analizan frecuentemente la eficiencia del proyecto; en criterios económicos son analizados los costos de inversión y costos de operación y mantenimiento; en criterios ambientales las emisiones de $\mathrm{CO}_{2}$ y uso del suelo, así como los impactos al ecosistema; y finalmente en la parte social son frecuentemente analizados la generación de empleo y la aceptación social.

Otros criterios comúnmente utilizados para evaluar los cultivos, y que serán considerados en el presente trabajo, son los siguientes:

\section{Balance energético}

Un método para evaluar la eficiencia energética de los cultivos es a través del balance energético, que comprende la relación entre la energía invertida en la producción del biocombustible (input) y la energía obtenida en su combustión (output). De acuerdo con Neto et al. (2004) el balance energético indica las viabilidades económicas y ambientales para cada tipo de cultivo.

El balance energético representa un componente esencial de varios tipos de evaluaciones ambientales, tales como balance ecológico, eco-auditorias o también en estimativas ecológicas ligadas a ingeniería (JORGENSEN y JORGENSEN, 1999).

Este balance puede, por lo tanto, ser una herramienta útil para optimizar el uso de insumos para determinado cultivo, incluyendo cultivos de invierno como la canola (Brassica napus L.). Lo anterior ha sido demostrado en diversos estudios de caso (JORGENSEN y JORGENSEN, 1999); (RATHKE y W. DIEPENBROCK, 2006)); (BUGGE, 2001). No obstante, diferencias en la escala, métodos y objetivos aplicados pueden dar paso a diferentes resultados entre los estudios.

Indicadores energéticos demuestran la eficiencia productiva de diversos sistemas, sin embargo, también 
permiten comparar diferentes productividades (HACISEFERIGULLARI et al., 2003) y son, por lo tanto, un adecuado complemento de análisis económicos (JONES, 1989).

\section{Rendimiento de extracción de aceite}

El proceso de extracción de aceites vegetales en escala industrial es realizado por:

- Extracción directa por solvente, como ejemplo el de la soya que presenta un contenido de aceite por debajo del $20 \%$.

- Extracción con pre-prensado seguida de extracción por solvente, como es el caso típico de la canola.

- Extracción por prensado, utilizada en frutos húmedos como la palma y almendras con alto contenido de aceite.

\section{Debito de carbono}

El creciente uso de energía, las alteraciones climáticas y las emisiones de dióxido de carbono $\left(\mathrm{CO}_{2}\right)$ de los combustibles fósiles priorizan el cambio para combustibles con bajo contenido de carbono. Los biocombustibles son una alternativa como potencial fuente de energía de bajo carbono, no obstante, debe considerarse la forma como son producidos. La conversión de florestas tropicales, turbas, sabanas o áreas para producir biocombustibles basados en cultivos alimenticios en Brasil, sureste asiático y en los Estados Unidos, genera una "deuda de carbono de biocombustible" al liberar 17 a 420 veces más $\mathrm{CO}_{2}$ que las reducciones anuales de gases efecto invernadero (GEI). Por otra parte, los biocombustibles producidos a partir de biomasa residual o de biomasa cultivada en terrenos agrícolas degradados y abandonados plantados con cultivos perennes presentan poca o ninguna deuda de carbono y pueden ofrecer ventajas inmediatas y sustentadas de GEI (FARGIONE et al., 2008).

Se considera "deuda de carbono" como la cantidad de $\mathrm{CO}_{2}$ liberada durante los primeros 50 años de este proceso de conversión de tierras. Con el tiempo, los biocombustibles de tierras convertidas pueden pagar esta deuda de carbono si su producción y combustión tienen emisiones de GEI menores que las emisiones del ciclo de vida de los combustibles fósiles que éstas remplazan. Hasta que la deuda de carbono sea reembolsada, los biocombustibles de las tierras convertidas tienen impactos (FARGIONE et al., 2008).

\section{Aplicación de glifosato}

Una de las características agrícolas intensivas de los últimos años refiere al uso de herbicidas como control vegetal en cultivos con el fin de maximizar rendimientos y beneficios económicos (ALLEGRINI et al., 2015). El glifosato [N-(fosfonometil) glicina] es un herbicida descubierto por Henri Martin en 1950, y actualmente es el más utilizado comercialmente en el mundo según EPA (2012) citado por (MESNAGE et al., 2015), y gran parte del crecimiento en su consumo se debe al uso de cultivos resistentes al glifosato tales como la soya, maíz y canola de acuerdo con Cerdeira et al. (2015) citado por (ALLEGRINI et al., 2015). El uso de este herbicida conlleva a diferentes efectos a largo plazo, tanto en la biodiversidad local (plantas, suelos, animales, cuerpos de agua), cuanto en la salud humana lo que ha producido un gran debate en su uso.

\subsection{Biodiesel}

Los ésteres monoalquílicos de ácidos grasos comúnmente llamado como biodiesel es un biocombustible liquido obtenido de diferentes fuentes renovables como aceites vegetales, grasas animales y aceites a partir de microalgas. Según Abunhabaya et al. (2012), las materias primas más utilizadas para la producción de biodiesel, son el aceite de girasol en la mayor parte de países europeos, aceite de soya en Estados Unidos y Brasil, aceite de palma en el sureste de Asia (Malasia e Indonesia) y aceite de coco en Filipinas.

\section{Metodos y materiales}

\subsection{Cultivos y criterios}

Uno de los objetivos del presente estudio es analizar los cultivos de canola, soya, palma y maíz para la 
producción de biodiesel de acuerdo con 3 categorías de evaluación que corresponden a aspectos técnicos, ambientales y económicos.

\subsection{Aspectos técnicos}

Representan las características productivas de cada cultivo. Se consideraron como inputs o criterios para el modelo el rendimiento de extracción de aceite, y balance energético (Tabla I).

El primer criterio representa la relación entre la cantidad de aceite producido del cultivo por el área cultivada. Según Pequeno (2010), el cultivo de palma supera en gran medida los demás cultivos con un rendimiento entre 3.0-6.0 toneladas por hectárea cada año. El aceite de soya, que es la principal materia prima de producción de biodiesel en el país con una participación del (69,2\%) (MME, 2015), posee un rendimiento de 0.2-0.6 ( $\mathrm{t} / \mathrm{ha}$-año). Por otro lado, el cultivo de maíz es el que presenta menor rendimiento de extracción de aceite con 0.18-0.36 (t/ha-año).

En el balance energético que representa la relación entre la energía invertida o consumida en la producción del biocombustible (input) y la energía obtenida en su combustión (output), son considerados los cálculos de las actividades relacionadas a la producción agrícola, transporte y procesamiento industrial.

En este criterio, para cada unidad de energía que entra al sistema, el cultivo que produce más energía es la palma con una relación de 1:4.6 (Mcal/ha), seguido del cultivo de soya con 1:3.95 (GAZZONI et al. 2005; BORGES et al., 2008 y GAZZONI et al., 2009). Para el caso del maíz, para una unidad de energía que entra al sistema, 0.77 (Mcal/ha) son producidas presentando la más baja relación de balance energético entre los cultivos analizados.

Tabla 1 - Rendimiento de extracción de aceite y balance energético

\begin{tabular}{ccc}
\hline Cultivo & $\begin{array}{c}\text { Rendimiento de extracción de } \\
\text { aceite (t/ha ano) }\end{array}$ & Balance energético (Mcal/ha) \\
\hline Canola & $0,5-0,9^{1}$ & $1: 2,9^{2}$ \\
Soya & $0,2-0,6^{1}$ & $1: 3,95^{3}$ \\
Palma & $3,0-6,0^{1}$ & $1: 4,6^{4}$ \\
Maíz & $0,18-0,36^{1}$ & $1: 0,77^{5}$
\end{tabular}

Fuente: 1- PEQUENO (2010); 2- GAZZONI et al. (2009); 3- GAZZONI et al. (2005); 4- BORGES et al. (2008); 5- PIMENTEL et al. (2005).

\subsection{Aspectos ambientales}

Esta categoría representa los impactos ambientales dados por el cultivo de estas oleaginosas tales como la aplicación de glifosato, la deuda de carbono y el uso del suelo para la producción de biodiesel.

El primer criterio considerado es la aplicación anual del componente activo del glifosato en los cultivos, que presenta grandes impactos producidos por este herbicida, además porque 3 de los 4 cultivos analizados en el presente estudio son cultivos resistentes al glifosato, favoreciendo a la aplicación de este herbicida y afectando el ecosistema local donde es aplicado. Los datos presentados en la Tabla 2 corresponden a la aplicación total anual de glifosato en los principales cultivos de los Estados Unidos y la respectiva área total cultivada durante el año de 2014 (BENBROOK, 2016; NASS, 2015). La cantidad del componente activo por hectárea cultivada de soya es de $1.64 \mathrm{~kg} / \mathrm{ha}$. Para el cultivo de maíz, la aplicación es de $0.85 \mathrm{~kg} / \mathrm{ha}$ y finalmente canola con $0.14 \mathrm{Kg} / \mathrm{ha}$.

Por otro lado, el cultivo de palma presentó los valores más altos con una aplicación del componente activo de glifosato de 2 kilos por hectárea según estudio realizado por Ikuenobe et al. (1998). La deuda de carbono consiste en el balance de emisiones de dióxido de carbono debido a la conversión de ecosistemas nativos 
para la producción de biodiesel mediante la implantación de monocultivos. Para este trabajo analizamos los datos encontrados por Fargione (2008), donde fueron considerados el biodiesel de soya producido en la amazonía brasilera, biodiesel de palma de la floresta tropical de turba en Malasia y el biodiesel de maíz producido en pastizales de los Estados Unidos. El cultivo de palma posee el índice más alto con 3452 miligramos de dióxido de carbono producidos por hectárea plantada (mgCO2/ha), seguido de la soya con 737 y finalmente el índice más bajo es del maíz con $134 \mathrm{mgCO} / \mathrm{ha}$. Los datos son presentados en la Tabla 2.

Finalmente, el uso del suelo corresponde a la relación del área cultivada necesaria para producir una tonelada de aceite de los diferentes cultivos. Esta relación representa una medida para un uso eficiente de la tierra cultivable. En la tabla 2 se presentan datos encontrados por Mourad (2006), donde la palma requiere de 0,5 hectáreas para producir 1 tonelada de aceite. Para el cultivo de soya y canola este valor es de 2.1 y 2.0 respectivamente. El cultivo del maíz por su parte presenta el mayor requerimiento de área para producir 1 tonelada de aceite con un valor de 4,7 (ha/t) presentando el uso menos eficiente entre los cultivos analizados.

Tabla 2 - Aplicación de glifosato, deuda de carbono y uso del suelo

\begin{tabular}{cccc}
\hline Cultivo & $\begin{array}{c}\text { Aplicación } \\
\text { glifosato }(\mathrm{kg} / \mathrm{ha})\end{array}$ & $\begin{array}{c}\text { Deuda de carbono } \\
\left(\mathrm{MgCO}_{2} / \mathrm{ha}\right)\end{array}$ & $\begin{array}{c}\text { Uso del suelo } \\
\text { (ha/t) }\end{array}$ \\
\hline Canola & $0,14^{1}$ & $\mathrm{ND}$ & $2^{1}$ \\
Soya & $1,64^{1}$ & $737^{2}$ & $2,1^{1}$ \\
Palma & $2.0^{3}$ & $3452^{2}$ & $0,5^{1}$ \\
Maíz & $0,85^{1}$ & $134^{2}$ & $4,7^{1}$
\end{tabular}

Fuente: 1- USDA, 2017; 2- FARGIONE, 2008; 3- IKUENOBE et al., 1998

\subsection{Aspectos económicos}

Como aspectos económicos se consideraron el costo de producción de los 4 cultivos analizados, así como el precio del aceite comercializado en los principales mercados internacionales.

Para este trabajo se tuvo en cuenta el costo de labores agrícolas que incluye: semillas, fertilizantes, defensivos agrícolas, maquinaria, operación con avionetas, mano de obra, gastos financieros, depreciación; costos fijos como: mantenimiento periódico y seguro del capital fijo y otros gastos como: asistencia técnica, transporte de producción, almacenamiento y gastos administrativos. Para fines comparativos se utilizaron los costos de producción en Brasil. Para el maíz y la soya se utilizaron datos de la zafra 2015/2016 en el estado de Mato Grosso, para la canola se utilizaron los datos de la zafra 2016/2017 en la región de Passo Fundo, estado de Rio Grande do Sul y para la palma se utilizaron datos de Belém, en el estado de Pará.

Según datos reportados por la Empresa Brasileira de Pesquisa e Agropecuaria-EMBRAPA y el Instituto Matogrossense de Economía Agropecuaria-IMEA, los costos totales de producción para el cultivo de canola, soya, palma y maíz son respectivamente 2391 (EMBRAPA TRIGO, 2016), 3378,82 (IMEA, 2016), 3415,60 (SANTOS et al., 2016) y 2729,11 (IMEA, 2016) reales por hectárea cultivada (R $\$ /$ ha) (Tabla 3).

Por otro lado, de acuerdo al departamento de agricultura de los Estados Unidos (USDA, 2017), el precio más alto de los aceites de los cultivos analizados fue el de canola comercializado en el mercado de Rotterdam a un valor de 899 dólares por tonelada métrica en noviembre del 2016. El precio del aceite de maíz y de soya en el mercado de los Estados Unidos para la misma fecha fueron de 812 y 761 \$USD/t respectivamente. Finalmente, en el mercado de Malasia el precio de la tonelada de aceite de palma estaba en 708 dólares (Tabla 3). 
Tabla 3 - Costo de producción de cultivos y precio del aceite

\begin{tabular}{ccc}
\hline Cultivo & $\begin{array}{c}\text { Costo de producción de cultivos } \\
(\mathrm{R} \$ / \text { ha) }\end{array}$ & Precio del aceite vegetal (U\$/t-métrica) \\
\hline Canola & $2391,00^{1}$ & $899^{5}$ \\
Soya & $3378,82^{2}$ & $761^{5}$ \\
Palma & $3415,60^{3}$ & $708^{5}$ \\
Maíz & $2729,11^{4}$ & $812^{5}$ \\
\hline
\end{tabular}

Fuente: 1- EMBRAPA TRIGO, 2016; 2- IMEA, 2016, 3- SANTOS et al., 2016; 4-IMEA, 2016; 5- USDA, 2017

\subsection{Análisis multicriterio}

Para estudio de multicriterio se utilizó la metodología MACBETH. Para el análisis de datos del modelo fue empleado el software M-Macbeth versión 3.0.0 creado por Carlos Bana e Costa, Jean-Marie De Corte y Jean-Claude Vansnick en 2005.

Se creó entonces la estructura del árbol con las 3 categorías mencionadas anteriormente (técnico, ambiental y económico) y para cada una de ellas se incluyeron nodos como los criterios (Figura 1) teniendo en cuenta una base de comparación entre las opciones y 2 referencias con valor máximo y mínimo de cada criterio.

Figura 1. Estructura del árbol y criterios (software M-Macbeth)

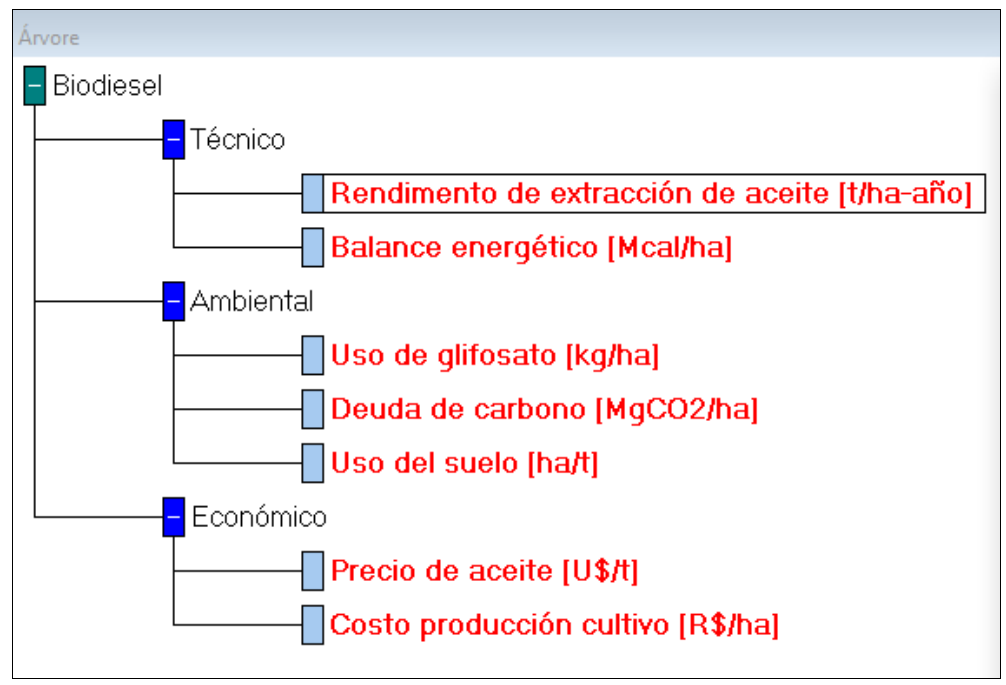

Posteriormente se realizaron las ordenaciones para cada criterio entre los 4 tipos de cultivos basándose en los valores presentados en las tablas de la sección anterior. Para el criterio "deuda de carbono", el decisor elaboró el siguiente ranking en orden creciente de atracción: canola, "neutro", maíz, "bueno", soya y palma.

A seguir, se le solicita al decisor que realice juicios cualitativos de la diferencia de atracción entre dos alternativas, entre ellas, debe seleccionar el nivel de preferencia de la alternativa canola con respecto al maíz, ya sea "extrema", "muy fuerte", "fuerte", "moderado", "leve", "muy leve" o "nula". El resultado de los cuestionamientos es ingresado en la matriz de preferencias del criterio en cuestión. La evaluación es repetida dentro de cada uno de los criterios evaluados.

El software M-Macbeth evalúa la consistencia de los juicios sugiriendo alteraciones en caso de haber inconsistencias. 
Luego de tener las ordenaciones consistentes para cada criterio, se analizaron las referencias de ponderación y se procedió a la ordenación de los pesos de los criterios, dando preferencia a los criterios ambientales con un peso acumulado entre sus criterios de los $52 \%$, luego técnicos con un peso de $28 \%$ y finalmente criterios económicos con $20 \%$.

Después de haber definido las preferencias dentro de cada criterio, es necesario establecer relaciones de preferencias entre los criterios, permitiendo el cálculo de una puntuación final para cada una de las alternativas.

De acuerdo con Bana e Costa et al. (2004) y Smith y Von Winterfeldt (2004), el procedimiento de evaluación intercriterios puede ser resumido en dos pasos:

- Paso 1: Considerando los niveles de "neutro" y "bueno" definidos para cada criterio anteriormente, se debe cuestionar el decisor de la siguiente forma: "imagine que exista una opción que sea neutra en todos los criterios establecidos. Cuanto aumentaría la atracción general de esta opción si pasa de "neutra" para "bueno" en el criterio $X$ ?

- Paso 2: El decisor emite juicios sobre la diferencia de atracción entre diferentes cambios posibles. Es decir, el decisor debe analizar qué tan atractivo es el cambio de "neutro" para "bueno" en el criterio "costo nivelado" que el criterio "costo de inversión". Nuevamente el decisor emite uno de los siete posibles juicios de valor (Figura 2).

Figura 2 - Juicios intercriterios (software M-Macbeth)

\begin{tabular}{|c|c|c|c|c|c|c|c|c|c|c|}
\hline W & & & & Ponderaci & on (Biodiesel) & & & & & \\
\hline 凅 & [Deuda de carbono] & [Rendimiento] & [Glifosato] & [Costo producción] & [Uso del suelo] & [ Balance energético] & [Precio aceite ] & [ all lower] & \begin{tabular}{l|} 
Escala \\
actual
\end{tabular} & extrema \\
\hline [Deuda de carbono] & nula & muy débil & muy débil & débil & débil & débil & moderada & positiva & 21 & m. fuerte \\
\hline [Rendimiento] & & nula & muy débil & muy débil & débil & débil & moderada & positiva & 20 & Tuere \\
\hline [Glifosato] & & & nula & muy débil & débil & débil & moderada & positiva & 19 & moderefacti \\
\hline [ Costo producción] & & & & nula & débil & débil & moderada & positiva & 17 & Imuy débil \\
\hline [Uso del suelo ] & & & & & nula & débil & débil & positiva & 12 & nula \\
\hline L Balance energético & & & & & & nula & débil & positiva & 8 & \\
\hline [Precio aceite ] & & & & & & & nula & positiva & 3 & \\
\hline [all lower] & & & & & & & & nula & 0 & \\
\hline
\end{tabular}

\section{Análisis de resultados}

Una vez realizada la ordenación de los pesos de cada criterio se obtuvo la tabla de puntuaciones para los 4 cultivos teniendo en cuenta los pesos de cada criterio, como ilustra las Tabla IV y V.

Tabla 4 - Tabla de rankings

\begin{tabular}{ccccccc}
\hline Rendimiento & $\begin{array}{c}\text { Balance } \\
\text { energético }\end{array}$ & Glifosato & $\begin{array}{c}\text { Deuda de } \\
\text { carbono }\end{array}$ & $\begin{array}{c}\text { Uso del } \\
\text { suelo }\end{array}$ & $\begin{array}{c}\text { Costo de } \\
\text { producción }\end{array}$ & Precio aceite \\
\hline Máximo & Máximo & Máximo & Máximo & Máximo & Máximo & Máximo \\
Palma & Palma & Canola & Canola & Palma & Canola & Palma \\
Sanola & Soya & Maíz & Maíz & Canola & Maíz & Soya \\
Maíz & Canola & Soya & Soya & Soya & Palma & Maíz \\
Mínimo & Maíz & Palma & Palma & Maíz & Soya & Canola \\
\hline
\end{tabular}


Tabla 5 - Tabla de puntuaciones, desempeño de las alternativas

\begin{tabular}{ccccccccc}
\hline Opciones & Global & Rendimiento & $\begin{array}{c}\text { Balance } \\
\text { energético }\end{array}$ & Glifosato & $\begin{array}{c}\text { Deuda de } \\
\text { carbono }\end{array}$ & $\begin{array}{c}\text { Uso del } \\
\text { suelo }\end{array}$ & $\begin{array}{c}\text { Costo de } \\
\text { producción }\end{array}$ & $\begin{array}{c}\text { Precio } \\
\text { aceite }\end{array}$ \\
\hline Palma & 46,78 & 100,00 & 100,00 & 0,00 & 0,00 & 100,00 & 22,22 & 100,00 \\
Maíz & 47,06 & 0,00 & 10,00 & 70,15 & 90,00 & 10,00 & 66,67 & 50,01 \\
Canola & 74,00 & 30,00 & 55,00 & 100,00 & 100,00 & 55,00 & 100,00 & 0,00 \\
Soya & 36,67 & 20,00 & 75,00 & 29,84 & 60,00 & 50,00 & 0,00 & 80,17 \\
[all upper] & 100,00 & 100,00 & 100,00 & 100,00 & 100,00 & 100,00 & 100,00 & 100,00 \\
[all lower] & 0,00 & 0,00 & 0,00 & 0,00 & 0,00 & 0,00 & 0,00 & 0,00 \\
\hline \multicolumn{2}{c}{ Pesos } & 0,20 & 0,08 & 0,19 & 0,21 & 0,12 & 0,17 & 0,03 \\
\hline
\end{tabular}

Se puede observar que el mayor puntaje global lo obtuvo el cultivo de canola con 74 puntos seguido del cultivo de maíz con 47.07 y palma con 46.78. En último lugar lo obtuvo el cultivo de soya con 36.67 puntos, que casualmente es el cultivo más utilizado en Brasil para la producción de biodiesel.

Es importante mencionar que a pesar del cultivo de palma obtener el primer puesto en 4 de los 7 criterios como lo muestra la Tabla 4 de rankings, fue el tercer colocado debido a sus muy bajos valores en criterios ambientales como aplicación de glifosato y deuda del carbono, que como se mencionó anteriormente, fueron los aspectos con mayor peso.

Por otro lado, el cultivo de canola obtuvo el primer lugar en los 3 criterios restantes siendo 2 de ellos aspectos ambientales mencionados anteriormente.

La toma de decisiones envuelve con frecuencia, información escaza, imprecisa o incierta. Puede ser útil por esta razón, analizar qué conclusiones robustas se pueden extraer del modelo para niveles variados de escasez, imprecisión e incerteza en la información utilizando la función "Análisis de robustez" de MMacbeth. Después de estas informaciones, se observa que no existe dominancia establecida entre los criterios (Figura 3). Luego, pequeños cambios en la opinión o preferencia del decisor pueden afectar el ranking de las alternativas.

Figura 3 - Análisis de robustez (software M-Macbeth)

\begin{tabular}{|c|c|c|c|c|c|c|}
\hline \multicolumn{7}{|c|}{ Análisis de robustez } \\
\hline 电 & Palma & Maíz & Canola & Soja & [ all upper ] [ & [ all lower ] \\
\hline Palma & $\equiv$ & $?$ & $?$ & $?$ & & $\Delta$ \\
\hline Maíz & $?$ & $=$ & $?$ & $?$ & & $\Delta$ \\
\hline Canola & $?$ & $?$ & $=$ & $?$ & & $\Delta$ \\
\hline Soja & $?$ & $?$ & $?$ & $=$ & & $\Delta$ \\
\hline \begin{tabular}{|l|} 
[ all upper ] \\
\end{tabular} & $\Delta$ & $\triangle$ & $\triangle$ & $\Delta$ & $\equiv$ & $\triangle$ \\
\hline [ all lower] & & & & & & $=$ \\
\hline \multicolumn{4}{|c|}{ Información local } & \multicolumn{3}{|c|}{ Información global } \\
\hline & ordinal & MACBETH & 1. cardinal & ordili & ial MACBETH & \begin{tabular}{l|l|}
$H$ & cardinal \\
& $\Gamma+0 \% * A$
\end{tabular} \\
\hline \begin{tabular}{|c|} 
Rendimiento \\
\end{tabular} & $\bar{v}$ & $\bar{v}$ & $\Gamma \pm 0 \%-$ & $\sqrt{v}$ & $\bar{\nabla}$ & $\Gamma \pm 0 \%$ \\
\hline 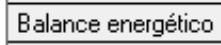 & $\bar{\nabla}$ & & $\Gamma \pm 0 \%=$ & & & \\
\hline Glifosato & V & V & $\Gamma \pm 0 \%=$ & & & \\
\hline Deuda de carbono & $\nabla$ & $\nabla$ & $\Gamma \pm 0 \% \div$ & & & \\
\hline Uso del suelo & $\nabla$ & $\nabla$ & $\Gamma_{ \pm 0 \%}=$ & & & \\
\hline Costo producción & $\nabla$ & $\nabla$ & $\Gamma \pm 0 \%-$ & & & \\
\hline Precio aceite & V & V & $\Gamma \pm 0 \% \vec{\nabla}$ & & & $w$ Diff \\
\hline
\end{tabular}




\section{Conclusiones}

Uno de los problemas que vive el mundo son las emisiones de gases efecto invernadero donde el sector de transporte es el principal responsable debido al uso de combustibles de fuentes fósiles.

Ante esta situación surgieron los biocombustibles, y a su lado el biodiesel como una alternativa para mitigar estas emisiones. No obstante, debido a la gran diversidad de materias primas para la producción del biodiesel, es necesario encontrar la mejor opción entre los principales cultivos de oleaginosas para su producción tales como la soya, maíz, canola y palma.

Para que el procedimiento sea más racional se utilizaron técnicas analíticas de apoyo a la decisión de varios criterios para elegir la mejor oleaginosa, entre las alternativas mencionadas anteriormente. La distinción entre la metodología multicriterio y otros métodos que ayudan en el proceso de toma de decisiones es el grado de incorporación de los valores subjetivos de los tomadores de decisiones en el desarrollo del problema considerado, lo que se analiza la misma alternativa de manera diferente de acuerdo con el juicio de valor que toman las decisiones en cada criterio y el modelo software M-Macbeth resulta como una herramienta que permite determinar la mejor alternativa teniendo en cuenta criterios de diferentes ámbitos.

Al aplicar este modelo M-Macbeth y la revisión de la literatura, se concluyó que el cultivo de canola resultó ser la mejor opción para la producción de biodiesel debido a los altos puntajes en criterios ambientales que tenían un mayor peso comparado a los criterios técnicos y económicos.

Otros cultivos como la palma presentaron buenos resultados en criterios técnicos, sin embargo, en aspectos ambientales sus puntajes fueron mínimos.

Finalmente, la soya fue el cultivo con puntajes más bajos en el presente estudio, siendo la fuente más utilizada para la producción de biodiesel en Brasil. En este sentido, se recomienda la diversificación o desarrollo de diferentes fuentes que sean más sustentables.

\section{Referencias}

ABUNHABAYA, R.; FIELDHOUSE, J.; BROWN, "The optimisation of biodiesel production by using response surface methodology an its effect on diesel engine," International proceedings of chemical, biological \& environmental engineering, Singapura, vol. 48, pp. 17-21, 2012.

ALLEGRINI, M.; ZABALOY, M. C.; GÓMEZ, E. V. Ecotoxicological assessment of soil microbial community tolerance to glyphosate. Science Total Environment, United Kindom, vol. 533, pp. 60-68, 2015.

ANDRADE, G. N.; ALVES, L. A.; ANDRADE, F. V. S; SOARES DE MELLO, J. C. C. B. Evaluation of Power Plants Technologies Using Multicriteria Methodology Macbeth. IEEE Latin America Transation, Somerset vol. 14, n. 1, pp. 188-198, 2016.

ANGUEBES, F.; CÓRDOVA-QUIROZ, A.; GRISELDA, C. B. J.; AGUILAR-UCAN, C.; CASTILLOMARTÍNEZ, G.; CERÓN-BRETÓN, R.; RUÍZ-MARÍN, A.; MONTALVO-ROMERO, C. Optimization of Biodiesel Production from African Crude Palm Oil ( Elaeis guineensis Jacq ) with High Concentration of Free Fatty Acids by a Two-Step Transesterification Process," no. January, pp. 13-21, 2016.

BANA E COSTA, C. A.; CHAGAS, M. P. A career choice problem: An example of how to use MACBETH to build a quantitative value model based on qualitative value judgments. European Journal Operation Resource, United Kingdom, vol. 153, pp. 323-331, 2004.

BANA E COSTA, C. A.; CORTE, J.-M.; VANSNICK, J.-C. M-MACBETH - Manual do Usuário. pp. 1-55, 2005. 
BENBROOK, C. M. Trends in glyphosate herbicide use in the United States and globally. Environmental Science Europe, Bethesda, vol. 28, no. 1, p. 3, 2016.

BORGES, J. L. B.; FELICI, P. H. N.; ÁVILA, M. T.; GAZZONI, D. L. Balanço Energético na Cultura do Dendê para Produção de Biodiesel - Parte Agrícola. in III Jornada acadêmica da Embrapa Soja, Documentos, E. Soja, Ed. Londrina: Embrapa soja, 2008, pp. 74-79.

BUGGE, J. Note: Rapeseed oil for transport 1: Energy balance and CO2 balance based on embio, the Danish energy agency's model for economic and enviromental assessment of biofuels. Hurup Thy, 2001.

CHAVES, M. C. C.; GOMES, C. F. S. Avaliação de biocombustíveis utilizando o apoio multicritério à decisão. Produção Online, Curitiba, vol. 24, no. 3, pp. 495-507, 2014.

COBULOGLU, H. I.; BÜYÜKTAHTAKIN, I. E. A stochastic multi-criteria decision analysis for sustainable biomass crop selection. Expert Systems with Applications, United Kingdom, vol. 42, n. 1516, pp. 6065-6074, 2015.

CRAGO, C. L.; KHANNA, M.; BARTON, J.; GIULIANI, E.; AMARAL, W. Competitiveness of Brazilian sugarcane ethanol compared to US corn ethanol. Energy Policy, United Kingdom, vol. 38, no. 11, pp. 7404-7415, 2010.

CUNHA, S. R.; ESPINOSA M. D.; KRAUSE, L. C.; MORAES, M. S. A.; FACCINI, C. S.; JACQUES, R. A.; ALMEIDA,; RODRIGUES, M. R. A. Beef tallow biodiesel produced in a pilot scale. Fuel Processing Technology, United Kingdom vol. 90, no. 4, pp. 570-575, 2009.

DE CASTRO, D. A. R. Estudo das variáveis operacionais dos processos de separação na produção de biodiesel a partir do óleo de palma bruto (Elaeis guineensis, Jacq) em escala de laboratòrio. Universidade Federal do Pará, 2013.

DIAKOULAKI, D.; KARANGELIS, F. Multi-criteria decision analysis and cost-benefit analysis of alternative scenarios for the power generation sector in Greece. Renewable Sustainable Energy Review, United Kingdom, vol. 11, no. 4, pp. 716-727, 2007.

EMBRAPA TRIGO, “Lucratividade na canola é discutida em curso - Portal Embrapa," 2016. [Online]. Available: https://www.embrapa.br/en/busca-de-noticias/-/noticia/11101764/lucratividade-na-canola-ediscutida-em-curso. Acesso em: 27 de julho de 2017.

FARGIONE, J.; HILL, J.; TILMAN, D.; POLASKY, S.; HAWTHORNE, P. Land clearing and the biofuel carbon debt. Science, vol. 319, no. 5867, pp. 1235-1238, 2008.

GAZZONI, D. L.; BORGES, J. L. B.; DE ÁVILA, M. T.; FELICI, P. H. N. Balanço energético da cultura da canola para a produção de biodiesel. Espaço Energia, Curitiba, n. 11, pp. 24-28, 2009.

GAZZONI, D. L.; FELICI, P. H. N.; CORONATO, R. M. E. S.; RALISCH, R. Balanço energético das culturas de girassol e soja para produção de biodiesel. Biomassa e Energia, Porto Alegre, vol. 2, no. 4, pp. 259-265, 2005.

HACISEFERIGULLARI, H.; ACAROGLU, M.; GEZER, I. Determination of the Energy Balance of the Sugar Beet Plant. Energy Sources, vol. 25, n. 1, pp. 15-22, 2003.

HARALAMBOPOULOS, D. A.; POLATIDIS, H. Renewable energy projects: structuring a multi-criteria group decision-making framework. Renewable Energy, United Kingdom, vol. 28, no. 6, pp. 961-973, 2003. 
IKUENOBE, C. E.; AYENI, A. O. Herbicidal control of Chromolaena odorata in oil palm," Weed Research, United Kingdom, vol. 38, no. 6, pp. 397-404, 1998.

IMEA-INSTITUTO MATOGROSSENSE DE ECONOMIA E AGROPECUÁRIA. Custo de produção de soja - safra 2016/2017. Cuiabá, 2016.

JONES, M. R. Analysis of the Use of Energy in Agriculture-Approaches and Problems. Agricultural System, vol. 29, pp. 339-355, 1989.

JORGENSEN, R. N.; JORGENSEN, B. J. N2O emission from miscanthus, rye and oilseed rape - In relation to the net CO2 -displacement. Frederiksberg, 1999.

KURKA, T.; BLACKWOOD, D. Selection of MCA methods to support decision making for renewable energy developments. Renewable Sustainable Energy Review, United Kingdom, vol. 27, pp. 225-233, 2013.

M. A. G. PEQUENO, "Avaliação do potencial produtivo de óleos obtidos a partir de microalgas por cromatografia gasosa," Universidade Federal da Paraíba, 2010.

MESNAGE, R.; DEFARGE, N.; SPIROUX DE VENDÔMOIS, J.; SÉRALINI, G. E. Potential toxic effects of glyphosate and its commercial formulations below regulatory limits. Food Chemical Toxicology, United Kingdom, vol. 84, pp. 133-153, 2015.

MINISTÉRIO DE MINAS E ENERGIA. Balanço Energético Nacional 2015: Ano Base 2014. Empresa Pesquisa Energética - EPE, p. 291, 2015.

MOURAD, A. N. Principais culturas para obtenção de óleos vegetais combustíveis no Brasil. Proc. 6. Encontro Energia no Meio Rural, p. 10, 2006.

NATIONAL AGRICULTURAL STATISTICS SERVICE (NASS), “Acreage,” pp. 1-5, 2015.

NETO, J. A. A.; CRUZ, R. S.; ALVES, J. M.; PIRES, M. M.; ROBRA, S.; PARENTE JÚNIOR, E. Balanço energêtico de ésteres metílicos e etílicos de óleo de mamona. I Congr. Bras. mamona-Energia e sustentabilidade, vol. 1, p. 7, 2004.

PIMENTEL, D.; PATZEK, T. W. Ethanol production using corn, switchgrass, and wood; Biodiesel production using soybean and sunflower. Natural Resources Research, Cham, vol. 14, n. 1, p. 65-79, 2005.

RATHKE, G.; DIEPENBROCK, W. Energy balance of winter oilseed rape ( Brassica napus L.) cropping as related to nitrogen supply and preceding crop. European Journal Agronomy, United Kingdom, vol. 24, n. 1, pp. 35-44, 2006.

SANTOS, J; HOMAA, A.K.O.; GOMES-JUNIOR, R. A.; SENA, A.L.S.; MENEZES, A.J.E.A.; MONTEIRO, K.F.G.; PEREIRA, R. Avaliação do Desempenho Econômico e do Potencial de Geração de Renda da Estrutura Produtiva de Pequena Escala de Dendezeiro Híbrido Interespecífico na Mesorregião Metropolitana de Belém. Belém, 2016.

SMITH, J. E.; VON WINTERFELDT, D. Decision Analysis in Management Science. Management Science, Bethesda vol. 50, n. 5, pp. 561-574, 2004.

STRANTZALI, E.; ARAVOSSIS, K. Decision making in renewable energy investments: A review. Renewable Sustainable Energy Review, United Kingdom, vol. 55, pp. 885-898, 2016. 
USDA-UNITED STATES DEPARTAMENT OF AGRTICULTURE. “Oilseeds: world markets and trade," 2017.

W. F. SAWIN J., SEYBOTH K., Renewables 2017 Global Status Report. 2017. 